\title{
Theory of Dynamic Interactions: Innovations
}

\author{
Alejandro Álvarez \\ Advanced Dynamics, C.B., Madrid, Spain \\ Email: gestor@advanceddynamics.net
}

How to cite this paper: Álvarez, A. (2017) Theory of Dynamic Interactions: Innovations. World Journal of Mechanics, 7, 101119.

https://doi.org/10.4236/wjm.2017.73010

Received: January 12, 2017

Accepted: March 26, 2017

Published: March 30, 2017

Copyright $\odot 2017$ by author and Scientific Research Publishing Inc. This work is licensed under the Creative Commons Attribution International License (CC BY 4.0).

http://creativecommons.org/licenses/by/4.0/

\begin{abstract}
The theory of dynamic interactions suggests a new paradigm of mechanics and initiates us into a new area of knowledge, hitherto undeveloped. In this paper, we describe the innovations that this theory brings to physics, and in particular, the ideas expressed on new book by Doctor Barceló: New paradigm in physics. It is necessary to analyse the incorporation into mechanics not only of knowledge about bodies with inertial movement, but also that of noninertial systems. It is necessary a new structure of knowledge that can incorporate both inertial and accelerated systems. On this paper we referred to the main innovations and novel ideas proposed by Doctor Barceló on his new book, in relation to the rotational dynamics.
\end{abstract}

\section{Keywords}

Rotational Motion, Dynamic Interactions, Rotational Inertia, Accelerated Systems, Angular Displacement

\section{Introduction}

Sometimes, in the scientific community, there are individuals who risk more than most, and suggest new intellectual horizons. Those who are heterodox take the path of what Kuhn calls revolutionary science, trying to find solutions to unresolved problems or ones that it has not been desired to rethink until that time, confirming the existence of a crisis that many others do not want to recognize, which usually leads to a conceptual framework that rivals the existing one. The new paradigm proposed usually generates great rivalry and, in its beginnings, may present anomalies, partly due to still being incomplete. Most of the scientific community will oppose any conceptual change, and it will take more than a generation of scientists, until that new paradigm is accepted.

For Doctor Barceló, physicist and engineer, author of the book: New Paradigm in Physics, classical mechanics applied to the rotation of rigid solids offers a series of doubts that force the revision of the assumed principles in the struc- 
tural development of the equations that govern and try to explain the movement of these. The text addresses these differences in depth and provides a novel theory for the explanation of these phenomena, TDI (Theory of Dynamic Interactions).

This text wishes to recall the innovations suggested in the book New Paradigm in Physics [1]. In this article, we discuss and deal with the points that are divergent with classical mechanics, as well as outlining TDI, its theoretical basis, and its main innovations.

\section{Initial Inquiries}

In his initial inquiries, our author warns of the existence of contradictions and possible inconsistencies in classic mechanics, especially when analyzing bodies in rotation.

The textbooks that he studies do not convince him and he observes how, with the spinning top [2], two simultaneous rotations can be considered: the one rotation around its axis and the other around the fulcrum. He verifies that this diagnosis is not referred to in textbooks. Even these books usually refer to a spinning top on a fixed point, when the characteristic of this toy is its dance, which for centuries has entertained children. He also warns that: The equilibrium we can observe in our universe and its dynamics did not seem to fit in with the conceptual structure of classical mechanics [3].

With these observations, Dr. Barceló began to analyze the behavior of bodies subjected to different simultaneous or successive rotations, both from a theoretical point of view, as well as through multiple experiments, being, errors accepted, the first researcher to explore this behavior, which is not described in classical mechanics. He himself states that: We have not come across beforehand, at least in the scientific literature of the 19th and 20 th centuries, any analysis or study similar to that of this text dealing with the dynamic phenomena that arise in systems or in solid rigid bodies subject to external actions that generate spatially non-concurrent, simultaneous acceleration fields. Accordingly, we understand this work to be hitherto unheard of and the conclusions arrived at never having been announced heretofore.

We shall present new dynamic hypotheses for accelerated systems in this text that we believe to have been unknown to date [4].

\section{Identifying Confusion and Caveats}

Firstly, he starts out by identifying contradictions and inconsistencies, which classical mechanics warns us of, arriving at the conclusion that they can be encompassed in a generalized group of incompatibilities, stating: Accordingly, we understand that the application of Newtonian mechanics to moving systems in non-inertial frameworks, returning conceptually erroneous results, would not pass a suitability audit [5].

In this way, he concludes that it is not possible to apply Newtonian mechanics to rotational dynamics: they are incompatible, since one refers to systems in 
uniform movement and rotational dynamics is necessarily accelerated. In his opinion, classic mechanics is fully valid in its natural application to uniform, inertial and non-accelerated movements, but rotational dynamics cannot be incorporated within it because it must comply with the laws of non-inertial movement.

\subsection{Error Identification}

In the first place, he focuses on mathematical models applied to the analysis of rotations and their physical reality; he considers that mathematical rotation should not be confused with dynamic rotation, since this applies to the real behavior of nature, precisely due to its inertia. Therefore, he suggests that specialized books should clearly differentiate between:

- Mathematical rotation

- Physical rotation

Also, for the latter, he does not consider their vectorial representation fortunate, or, consequently, the application of vector algebra to rotations. He studies angular displacements, and concludes that they are not commutative, nor obey the law of vectorial addition [6].

For these reasons, he proposes that: There is no problem in understanding the commutability of coaxial rotations, but it is difficult to understand that any other group of non-coaxial rotations are commutative, when angular displacements are not, yet they do obey this law when they are infinitesimal. Indeed, it is even more difficult to understand how a finite number of infinitesimal displacements turn finite angular displacements into commutative ones.

Given this apparent contradiction, it seems rather risky to establish a rotational mechanics on the hypothesis of the vectorial behavior of angular velocities. Moreover, in our opinion, all of this means that it is doubtful to predict the situation of a solid subject to multiple rotations by means of an algorithm like the one developed by Euler or by vectorial mechanics.

Furthermore, the non-commutability of finite rotations of the bodies, and therefore, the failure to obey vectorial algebra, can be checked by simple experimental tests [7].

In the light of the foregoing, in our opinion, rotation dynamic magnitudes cannot be represented by vectors, nor vector algebra applied to these magnitudes [8].

The assumption of this mathematical commutativity of rotations, is at the base of the development of main equations of motion of solids in rotation.

\subsection{Euler's Theorem of Rotation}

A paradigmatic example of this confusion is, in geometry, the so-called: Euler's Theorem of Rotation. In reality, this is a mathematical theorem stating that in a three-dimensional space, any transformation by rotation that maintains a constant point must also leave an entire axis constant. Mathematically speaking, the combination of rotations constitutes another rotation, thus the set of rotation 
operations has an algebraic structure known as a group (...). Specifically, the group of rotations is defined as the "special orthogonal group or rotation group $S O(3)$ ". In mathematical terms, any composition of rotations on a solid rigid body with arbitrary axes is equivalent to a single rotation on a new axis.

However, these geometrical conclusions cannot be translated to dynamics without applying the scientific method, conducting the apposite trial and test. Without due rigor, Euler's theorem of rotation has been extended to dynamics, stating it as follows. Any rotation or group of successive rotations can always be expressed as a rotation around a single direction or main rotation axis. In this way, every rotation (or group of successive rotations) in three-dimensional space can be specified through the equivalent rotation axis defined vectorially by three parameters and a fourth one representative of the rotated angle [9].

Confusion about the rotational behavior of mass has been a constant throughout the history of physics, and this is a paradigmatic example of such confusion, leading the same author to claim that: According to Euler's rotation theorem, rotation around more than one axis at the same time is impossible, consequent$l y$, if two rotations are forced at the same time on a different axis, a new axis of rotation will appear. (Reference the same as the previous one).

The mathematical concept of rotation must not be mixed up with rotational dynamics, in which inertia establishes the true behavior of the mass. For example, in nature we find numerous examples of the existence of two simultaneous rotations on two different axes, without any new axis appearing on account of the coupling of the previous two. The boomerang, the spinning top and the gyroscope are all cases in point [10].

\subsection{Lack of Consistency}

But this incoherence was not the only caveat that could be posed to the rotational dynamics of classical mechanics, since the concept of the velocity coupling in motion is also added.

Nothing that his observations did not seem to coincide with the numerical results of orthodox formulations, he sought an objective third-party opinion. In the latter, motion equations were carried out in accordance with the Newton-Euler formulation, the prediction of the behavior of the submarine experiment was performed by Advanced Dynamics [11], and variational solutions with the equations of Lagrange and Hamilton were also developed. In all cases the report assured that moving object would maintain a straight path, generating a new rotation of the body, as a result of the vectorial addition of both existing rotations.

However, it was evident that the actual result observed was quite another. This proved that the equations were not suitable in the stated case. For this reason, Doctor Barceló suggests: The lack of consistency between the calculations done by third-party experts, in accordance with the normal criteria of analytical mechanics, and the results observed in the experimental tests, attest to the fact that the mathematical formulation of classical mechanics was not consistent with the 
real behavior of nature. Indeed, this contradiction even arose in the three calculation procedures applied [13].

But it was difficult to understand why this experimental verification had not been carried out before: It is strange to think that these types of experimental tests had not been conducted in the over two-hundred years since Euler had put forward the first calculation procedure. And even presumptuous to suppose that we were the first to detect this lack of fitness between the mathematical formulation and the observed reality [14].

The model of the Theory of Dynamic Interactions analyzes the interactions between rigid translational solids subjected to their own rotation and the interactions that external torques generate throughout their trajectory. The author incorporates in New Paradigm in Physics, figures, videos and animations to explain his dynamic theory.

In the case of bodies endowed with intrinsic angular momentum, the rotation of the body around an axis of symmetry will avoid the compounding of rotations. If a new torque acts on an axis that is spatially non-concurrent with the existing rotation, this will be kept up. And the body will react by modifying its path, adding a precession or orbital movement in space to the initial rotation, as if it seemed to be subject to a central force.

We claim that the body, instead of taking a linear path, as would be expected from the Newton-Euler equations in classical mechanics (Trajectory I in Figure 1), will take a curved path, as we have repeatedly said that we observed in our experimental tests, on account of the coupling of the resultant velocity fields on each point of the body (Trajectory II in Figure 1) [15].

Initially, Dr. Barceló warned that these formulations were unsolvable, so they did not allow an exact solution, and required an approximate resolution. This was an indication of the lack of suitability, since it was hardly credible that nature could behave without an exact predictable outcome.

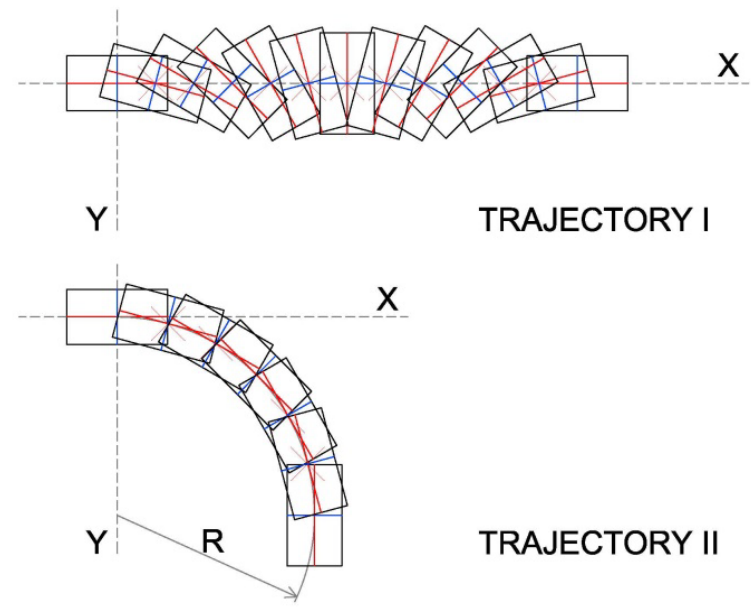

Figure 1. Trajectory I predicted by classical mechanics and trajectory II deduced by means of the Theory of Dynamic Interactions (TDI). We have observed and confirmed the second case repeatedly by experiment [12]. 
Parallel to the evolution of the conceptual analysis of rotation movements, its mathematical resolution tries to find solutions in the equations proposed by Euler, in which it is argued that the sum of momentums will determine the orientation movement of the mobile object, independently of the effect of the forces that simultaneously act upon it. According to these classical working hypotheses, the orientation of a rigid solid body can be determined from Euler's angular coordinates.

If these coordinates are known depending on time, we will be able to deduce the temporal evolution of its orientation. These are Euler's equations, based on which we can determine, at least conceptually, the trajectory of a body subjected to multiple momentums.

It was Euler who, according to Appell... presented, in their definitive form, the movement equations for a solid rotating around a fixed point. Indeed, he was also the first to come up with rigorous integrals for those cases in which the external forces were null or allow for a single resultant on passing through a fixed point [16], as attested to in the Reports of the Berlin Academy for 1758.

But we have to be aware that the system of differential equations that he proposes is not generally integrable, so it was not possible to have a generalized resolution of these equations, solving them only in concrete cases, in which the sum of the external forces was null or would admit a single result that passed through a fixed point. Still today, this same system of equations is used to determine the orientation of a solid subjected to multiple pairs [17].

For Dr. Barceló, this formulation based on commutative vector algebra is not applicable to physical rotations of matter in space, nor is the computational procedure which is still used today. He demonstrates his argumentation with the experimental tests that he carries out, and as a result, he says: After our analyses, we pointed to the inconsistency in the accepted Newton-Euler equations in their application to different bodies subject to diverse non-coaxial rotations [18].

\subsection{Obsolete Approximate Resolution Procedure}

In addition, the report confirmed how the experts were still using the approximate resolution procedure... suggested by Sofia Kovalévskaya and which saw her obtain the Bordin Prize from the French Academy of Sciences in 1888. Her numerical solution is still applied nowadays, in the computer era, for these unsolvable equations, an approximate calculation procedure based on a supposed conceptual development [19].

For all this, the author adds in his Epilogue: It is also a source of wonder to witness how that accepted calculation procedure determines a path for these bodies that does not concur at all with that which can be observed in nature. This lack of consistency between the result of the formulas applied and the real nature of the observed path, makes us think that in all these years no experimental tests or checks have been done to confirm whether or not these algorithms reflect reality or were simply mathematical structures unconnected with the truth of nature. 
Therefore, we repeat that a profound examination of the procedures is needed, and even a revision of the principles of classical and analytical mechanics for these non-inertial cases with a view to the results of the equations in question being adapted to the observable reality of moving objects in nature subject to accelerations [20].

We can add that it is quite possible that Euler did not become aware that, in the field of the mechanics of non-inertial systems, he was incorporating inertial concepts to develop his equation of motion of bodies with intrinsic rotation. Something we have already defined as incompatible, since bodies subjected to rotations, are supposed to non-inertial.

But in addition to this conceptual error, he does not seem to check the results of his formulation with actual experiments.

However, it is even worse, if the scientific consensus on this formulation was maintained, without its falsifiability or verification for so many years.

\section{TDI Innovations}

In addition to identifying errors and caveats, the author proposes new equations for accelerated rotational movement, and other multiple conceptual and technological innovations for accelerated systems.

The innovative approach of Dr. Barceló will allow broad scientific and technological progress in non-inertial dynamics.

\subsection{A New Celestial Mechanics}

The development of the theory has allowed its author to conceive a new model of celestial mechanics. In the book, it says: The hypotheses and axioms proposed fully explain the doubt or initial aporia inherent in the fact that celestial bodies in the observable universe rotate and orbit simultaneously, not because of causality, but rather owing to a defined, logical and regulated behavior. Moreover, the rational structure that is proposed provides explanations about the behavior of celestial bodies in general, not to mention the fact of enabling us to justify other more dynamic phenomena that have scarcely been understood to date, such as, for example, the dance of the spinning top or the flight of the boomerang [21].

This new vision of the universe was already set forth by Dr. Barceló in his book: Imago Universi [22] and in his article: Proposal of New Criteria for Celestial Mechanics [23], and will be developed more extensively in chapter VIII of volume II.

\subsection{Immutable Dynamic Balance}

One of the features of this new conception of the dynamics of the cosmos is its constant and enduring balance: The Theory of Dynamic Interactions holds that a body with simultaneous translation and rotation movement, submitted to a new non-coaxial constant torque, will, instead of undergoing acceleration in its rotation as classical mechanics put forward, maintain a constant and apparently 
immutable dynamic balance. We consider that the theory we put forward, allows a better comprehension of the dynamic behavior of the celestial bodies and its age-old dynamic balance. Besides, we consider that this balance of the universe is not within the parameters of the present laws of classical mechanics [24].

\subsection{Flat Celestial Systems}

If we look closely at the universe, we see that it is made up of flat celestial systems. Galaxies, solar systems like ours, Saturn's rings, the asteroid belt, the Kuiper belt or the scattered disc have flat structures.

Precisely, the TDI's dynamic model justifies the universe's flat image, in which galaxies and systems also tend to develop into flat structures.

\subsection{Fictitious Forces}

Dr. Barceló conceives a pyramidal structure of mechanics, in which, from certain axioms, it is possible to deduce the behavioral laws and propose developmental theorems of the discipline. The structure of current classical mechanics does not take this conceptual scheme into account, to which effects and phenomena have been added, which have not been deduced from its initial axioms. In his opinion, this was a new indication of its structural inconsistency. In this area, so-called fictitious forces, deserve special mention, which are added in order to justify the behavior of moving object, which initial axioms cannot explain: The historical procedure of adding to dynamic contexts that purportedly fail to comply with the laws, with fictitious forces would not be open to a control audit and, thus, is not a good example of good mechanical knowledge management [25].

Adding later: The idea is not incorporate these fictitious forces into the structure of mechanics, but rather to come up with new orders of thought for rotational dynamics, ones that differ to those accepted to date for translational mechanics, and to analyze the origin of these singularities that we call inertial forces, looking for their origin in nature and proposing their dynamic reinterpretation by means of new laws of behavior that authentically, one-to-one, correspond to the true dynamic behavior observed in experiments.

We do not believe it necessary, as far as the logical structuring of dynamics is concerned, to incorporate these ideas of fictitious forces to understand the true dynamic behavior of bodies. Throughout this book we will explain the true behavior of nature without any need to introduce fictitious forces, or other ideas based on assumptions instead of observation [26].

\subsection{Adding Angular Momenta}

On observing the behavior of moving objects in rotation, when they are exposed to new non-coaxial rotations, Dr. Barceló reaches the conclusion that angular movements of non-coaxial momenta do not necessarily couple: This behavior of nature can also be interpreted in the field of physics by the fact that, under such circumstances, angular momenta are not added up, so if a body is has an angular momentum $L$, its variation $d L$, due to effects of external forces, will not neces- 
sarily be added to the already existing $L(\ldots)$, but rather can generate a new movement, different and simultaneous to the existing one, which we will call precession movement. This would be the case of the spinning top and the gyroscope, and in the case of a body in space without any constraints, like a boomerang, the body will begin to orbit without the necessary existence of a central force [27].

In this way, caveats and errors being identified, he conceives a new mathematical model to understand this natural behavior: The importance of our mathematical model is obvious. In this model not only the forces are leading players, but also the momenta of those forces which, while staying constant, will generate orbits and constantly recurrent movements, generating a system in $d y$ namic balance, and not in unlimited expansion. This new dynamics theory will give us a better understanding of how the universe and matter behave.

We would suggest a detailed and in-depth analysis of these dynamics related hypotheses and propose continuing the experimental testing necessary for confirmation [28].

\subsection{Rotational Model}

The initial dynamic hypotheses were confirmed with the experimental tests performed. It was confirmed that simultaneous rotations on different axes could coexist in the same moving object, and inertial reactions were generated: It proves necessary to incorporate (...) inertial reactions in general, into a logical and scientific structure in the field of mechanics [29].

The velocity fields determine the behavior of the body. In Figure 2 we proposed a comparative analysis of the velocity fields generated in a rotation and subjecting the body to two successive, non-coaxial torques. But, in addition, the coexistence of different rotations in the same body, generates this dynamic behavior, which to date had not been so clearly explained: Accordingly, the variation of the relative position of the particles on account of a new rotation on an axis other than the existing rotation axis, generates a variation in the linear velocity of the body, which is not uniform for all the disc particles located on the same radius. This instantaneous variation creates a distribution of accelerations on the disc that causes the appearance of the effective inertial forces, which are made evident as a dynamic interaction momentum [30].

This is precisely the so-called gyroscopic momentum of classical mechanics, but in the theory proposed by Dr. Barceló, it appears as a result of a rational deduction, and is integrated into the structure of the theory itself. In this way, it replies to Bruhat, who referred to the "Gyroscopic Effect" of paradoxical appearance [31].

Dr. Barceló develops his thinking in the area of the Field Theory, and raises the hypothesis, against the criterion established by Poinsot, that a coupling of fields of dynamic magnitudes of differentiated origin is generated (See Figure 3): In our experiment we confirmed what we had intuited in the simulation, namely that, the translational velocity field of the submarine couples with the velocity 


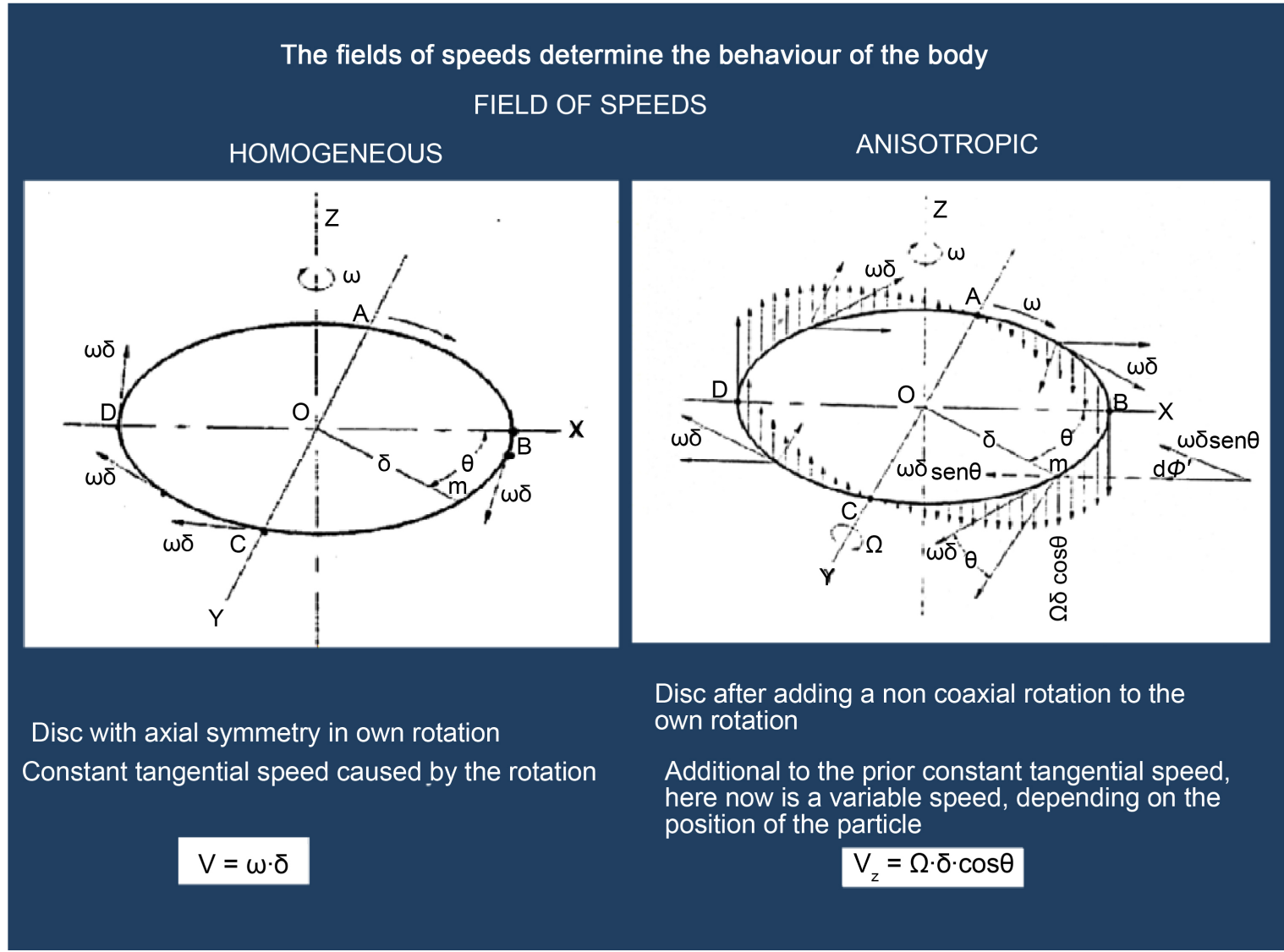

Figure 2. The velocity fields determine the behavior of the body. Comparative analysis of the velocity fields generated in a rotation and subjecting the body to two successive, non-coaxial torques [32].

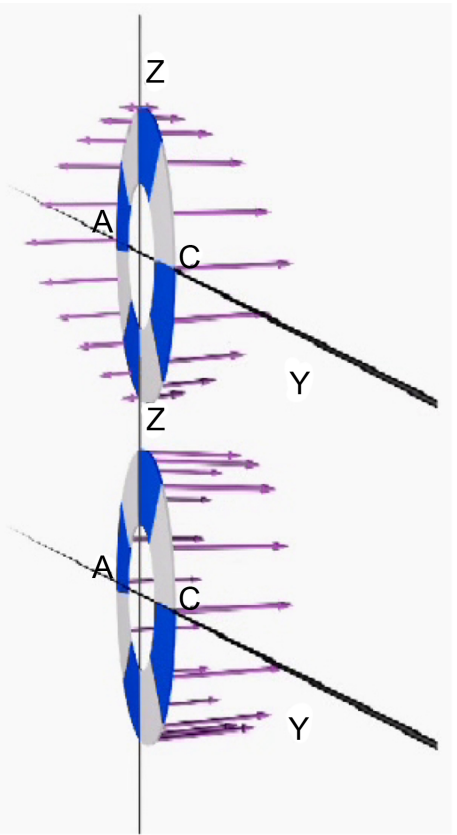

Figure 3. Velocity coupling: The $V_{c}$ anisotropic velocity field generated by the secondary torque (Top figure) dynamically couples with the translation velocity field $V_{T}$ (Bottom figure), thus generating a new velocity field $V_{R}$, which determines the curved path of the moving object [33]. 
field generated by the torque caused by weight and buoyancy vector. The result was the circular path observed in prototype I, as further attested to by the flight of the boomerang and the peculiar dance of the spinning top [34].

But he adds that this dynamic behavior of bodies accelerated by rotations is due to a defined mathematical model: the derivative of the cosine of an angle is equal to at least the sine of the same angle by the derivative of its angle, where this derivative is the angular velocity of the body on the initial axis [35].

After this analysis, he suggests: Accordingly, by means of an analysis of the velocity fields generated, the behavior of bodies accelerated by non-coaxial rotations and the ratio of the inertial reactions that are caused in matter in these cases has been clearly explained. Dynamic interaction phenomena, or phenomena with the gyroscopic effect as classical mechanics would have it, are fully explained by this analysis of the fields generated.

We believe that this dynamic description of the phenomenon analyzed truly reflects the behavior of nature and helps to clearly explain the inertial reactions of the mass of bodies [36].

To sum up, it can be affirmed that it would be possible in space for those bodies with angular momentum, subject to non-coaxial torques, owing to inertial dynamic interactions, to start a movement in such a way that, if the initial angular momentum is maintained and the second torque remains constant, an orbital path will hold up without any need for central forces [37].

\subsection{Angular Displacement}

Once a new physical-mathematical model is conceived, and experimentally verified, Dr. Barceló recalls that the actual behavior of nature paradoxically does not match the mathematical results obtained. This apparent contradiction can be reconciled if it is acknowledged that the formulations of mechanical classics have started from certain assumptions which, in our opinion, do not correspond to the real dynamic behavior of bodies in nature: This apparent contradiction can be reconciled if it is acknowledged that the formulations of mechanical classics have started from certain assumptions which, in our opinion, do not correspond to the real dynamic behavior of bodies in nature.

1) It is supposed that the movement of the center of gravity and the body's rotary movement decouple,

2) It is supposed that the existing angular momentum and the one generated by the new torque will couple;

3) It is supposed that rotating bodies and, therefore, the equations for rotational dynamics obey vector algebra.

Accordingly, in our opinion, these baseline hypotheses of classical mechanics need to be revised and operators incorporated into the mathematical calculation that determine the real inertial behavior of nature, and which will enable the appropriate mathematical algorithm to represent the phenomenon of dynamic interactions [38].

And these specifications, in the case of bodies subject to acceleration by rota- 
tion, suppose that:

- There is no composition of rotational movements in accordance with vector algebra.

- The principle of proportionality between forces and accelerations is not obeyed in the case of non-coaxial torques.

- Magnitudes of rotation, made up of the angle and its derivatives, are not added together algebraically.

- Neither does the commutative law of vectors apply.

- Discrete coupling occurs between the translational movement and that generated by the inertial reaction of the resultant interaction of the second torque.

- The initial intrinsic angular momentum does not couple with the resultant of other later actions.

- The reactions of the interaction generated by successive non-coaxial rotations must be incorporated into the mathematical formula.

- There is no need to use reference systems external to the body.

- The resulting mathematical equations will hold for a particular initial dynamic state and for a specific excitation, the results of which may vary depending on previous dynamic states or the excitations that arise.

- The dynamic state of a single body can be determined by logical sequences, which are open to representation by computer programs [39].

Adding that: Under certain circumstances, the constant rotation of a body on a main axis of its inertia ellipsoid prevents the compounding of rotations, in such a way that if a new torque acts with a non-concurring axis, this will hold up, and the body will react modifying its path, adding a precession or orbital movement in space to the initial rotation, as if it was apparently subject to a central force.

It is also important to reiterate the fact that the evolution of the dynamic state of a body is not determined by a single equation, but rather by logical sequences that can be represented by computer programs, given that the result of a given momentum, not only depends on the baseline parameters, but also on the type of excitations, their sequence and the already existing movements [40].

Dr. Barceló neither accepts the dimensional treatment reserved by classical mechanics for angular displacements, which has the following peculiarities:

1) Does not obey the algebraic law of vector addition.

2) Does not obey the commutative law.

3) Will obey the addition operation, but the result will depend on its priority in time.

4) It may be that the only algebraic operation of that magnitude with itself is addition.

5) For derivative operations, the following specifications should also be taken into account when acting on a solid:

- If $d A / d t(\mathrm{~A}=$ angular displacement $)$ is other than zero, the addition of a new derivative of this magnitude will not be added up algebraically, thus generat- 
ing an independent reaction, unless their directions coincide.

- If $\vec{L}$ is the intrinsic angular momentum of the solid body and $\vec{M}$ the momentum of a new torque that is applied, we get. $\vec{M}=\frac{\mathrm{d} \vec{L}}{\mathrm{~d} t}$, Where $\vec{L}$ and $\Delta \vec{L}$ are independent, not generating the addition of both, acting without coupling, unless their direction coincides.

- If $d A / d t$ is other than zero, the action of a torque $P \vec{P}$, made up of two equal forces $\vec{F}$ on the solid, with a translation velocity of $\vec{V}$, a displacement occurs on the body axis in the direction of the torque perpendicular to $\vec{F}$ instead of following its direction, thus modifying the direction of the vector $\vec{V}$, but not its module.

- The action of $\vec{P}$ causes a constant variation in the direction of velocity $\vec{V}$. If the action of $\vec{P}$ stops, the variation in the direction of the velocity $\vec{V}$ stops immediately [41].

Adding that: Moreover, we cannot understand how a rotational mechanics can be conceived without defining the angular displacement magnitude [42].

\subsection{Dynamic Interaction Inertia}

Throughout the text, Dr. Barceló incorporates new concepts and necessary terms in his structuring of mechanics. It is possible that rotational inertia might be one of the more interesting terms and he establishes it in this way:

1) Physics. Inability of rotating bodies to alter their dynamic state.

2) Physics. In a rotating body, it is the product of its mass by the square of its distance to the axis of rotation.

3) Inertia of the body when put in rotation on a main axis of inertia [43].

From these definitions, he applies the concept of rotational inertia: Consequently, in the bodies in which the dynamic interactions are caused, we find certain peculiar and distinguishing characteristics in their behavior, enabling us to refer to a dynamic interaction inertia, which can be defined as the tendency that all rotating bodies have to continue turning on the same plane and on the same axis. This behavior of the bodies has also been defined as rigidity in space.

Rigidity in space is a quality known in classical mechanics as a consequence of Newton's first law of motion and by applying the concept of inertia to rotating bodies.

Moreover, as we have already stated, bodies endowed with angular momentum can attain dynamic equilibrium, in such a way that, in the case of a gravitational field, as we have already pointed out, an energetic equilibrium will be caused between the difference of the potential energies of the solid, from the start until its equilibrium position, and its precession kinetic energy.

This behavior of matter enables us to define, in bodies endowed with intrinsic rotation, a rotational invariance, as a characteristic by means of which it can achieve a dynamic and energetic equilibrium, apparently immutable and constant on account of its inertial behavior.

The aforementioned rotational invariance can be understood as an intrinsic 
characteristic, independent of any reference system [44].

\subsection{New Hypotheses}

Adding later: Our research project arose from physical observation, the search for a dynamics for accelerated systems and from reflecting on the validity of classical mathematical models that accept the application of vector algebra to angular magnitudes:

- The mathematical model to develop classical equations of motion accepts vector algebra in spite of the fact that the magnitudes of rotational dynamics are neither commutative nor associative.

- Since Poinsot, classical mechanics has accepted the discriminant principle of the non-superposition of translation and rotation movements, and that the effects of forces do not couple with the effects of torques, without this reasoning ever having been checked experimentally [45].

\subsection{New Equation of Motion}

Based on the axioms put forward in his deductive process (see Figure 4), Dr. Barceló came to a new formulation for the movement of accelerated systems.

The new equation of motion makes it possible to learn of the true and real behavior of nature for non-inertial systems. It can be understood as a scientific realization that explains and predicts the behavior of accelerated dynamic systems. It provides new behavior models, thus solving dynamical problems [46].

\section{Specific axioms:}

1. The rotation of space determines the generation of fields.

We can observe in a body two intrinsic non-coaxial simultaneous rotations. In this case, the rotation of space determines the generation of anisotropic speeds and accelerations fields.

\section{Result of the action of non-coaxial moments}

When a solid is subjected to non-coaxial moments, non-homogeneous distributions of speeds and accelerations are generated. These can be identified as inertial fields.

\section{Inertial fields cause dynamic interactions.}

The anisotropic speeds fields generated, interact with other fields of the rigid body, changing its dynamic state. The non-homogeneous velocity field that is generated is coupled to the field of translation speeds.

\section{Successive actions of non-coaxial torques on a rigid body}

Successive actions cannot be determined by algebraic addition or calculated by the resultant force or torques.

\section{Field coupling}

The velocity fields generated by torques can be coupled with translation velocity fields.

Figure 4. Specific axioms of rotational dynamics [47]. 
The equation of motion finally turns out to be as follows [48]:

$$
\vec{V}=\overrightarrow{\vec{\Psi}} \vec{V}_{0}=\left(\begin{array}{ccc}
\cos M^{\prime} t / I \omega & -\operatorname{sen} M^{\prime} t / I \omega & 0 \\
\operatorname{sen} M^{\prime} t / I \omega & \cos M^{\prime} t / I \omega & 0 \\
0 & 0 & 1
\end{array}\right) \vec{V}_{0}
$$

\section{Structuring Mechanics}

At the end of the first volume, Dr. Barceló proposes a new structuring of mechanics: All types of mechanical knowledge of the solid rigid body should be organized and structured in accordance with a unified criterion for a better overall understanding and more fitting and efficient use, thus avoiding the incorporation of fictitious or non-real concepts to describe the observed manifestations, as well as preventing the incorporation of apparently anomalous phenomena.

(...) Notwithstanding, we do not have a conceptually defined, logical structure of the knowledge of non-inertial dynamic systems, nor have the laws of behavior for these cases been defined or ordered.

However, in nature, systems in motion that are subject to actions that generate accelerations are the norm, whereas inertial, dynamic systems are the exception. Specifically, systems subject to accelerations, and particularly, to acceleration by rotation, are normal in nature.

Scientific consensus has accepted that classical mechanics has developed as a paradigm of a specific discipline, when in truth, this should be a speciality of a much broader knowledge structure.

We believe that we should be more ambitious and generalist and try to initiate the development of the order of a general theory of mechanics, which refers to any observable type of mechanical state or movement, in either inertial or non-inertial conditions [49].

The author proposes the incorporation into mechanics not only of knowledge about bodies with inertial movement, but also that of non-inertial systems: We have set the foundations to a new structure of knowledge by putting forward a general theory of mechanics that can incorporate both inertial and accelerated systems, suggesting that a general theory of dynamic interactions be reserved to cover such cases.

We have also put forward a theory of dynamic interactions as a new paradigm for non-inertial dynamic systems with axial symmetry, which we believe makes it possible to better and more accurately predict the behavior of moving objects in such cases [50].

\section{Holistic}

Dr. Barceló also notes the consistency of mathematical results obtained in variational analysis, coincident with the Newton-Euler Equations, but not compatible with the trajectories observed in the experimental tests.

This incompatibility of the results of the formulations caused him great stupor, nevertheless, he deduced the origin of this new error in the formulation. The author thus states: It could be put forward that, in any of its formulations, 
the analysis of analytical mechanics is holistic, which means that its conception is based on the full and total integration of the phenomenon. Motion is not analyzed in accordance with temporal sequences or independent actions that provoke it, neither are its possible interactions or synergies taken into account in this analysis. In the light of the foregoing, on not considering inter-dependencies or possible interrelations in its development, we believe that this analysis should be revised [51].

Adding later: In our opinion, mathematical models cannot be developed on the basis of the total and overall integration of the movement that is analyzed. In their deduction, it is necessary to include all the parameters that impact on this behavior and to reason the supposed causes of that result. All interdependencies and possible interrelations that generate the particular path must be considered. Furthermore, the mathematical model obtained must be checked by experiment and test [52].

\section{Simulation Model}

In this case, that moving object's route is represented for a variable tangential velocity, the resultant path is not closed in this case, as can be observed in Figure 5 , describing as it does an orbital path. When the body is subject to a constant decelerated velocity, the path will describe a downward spiral.

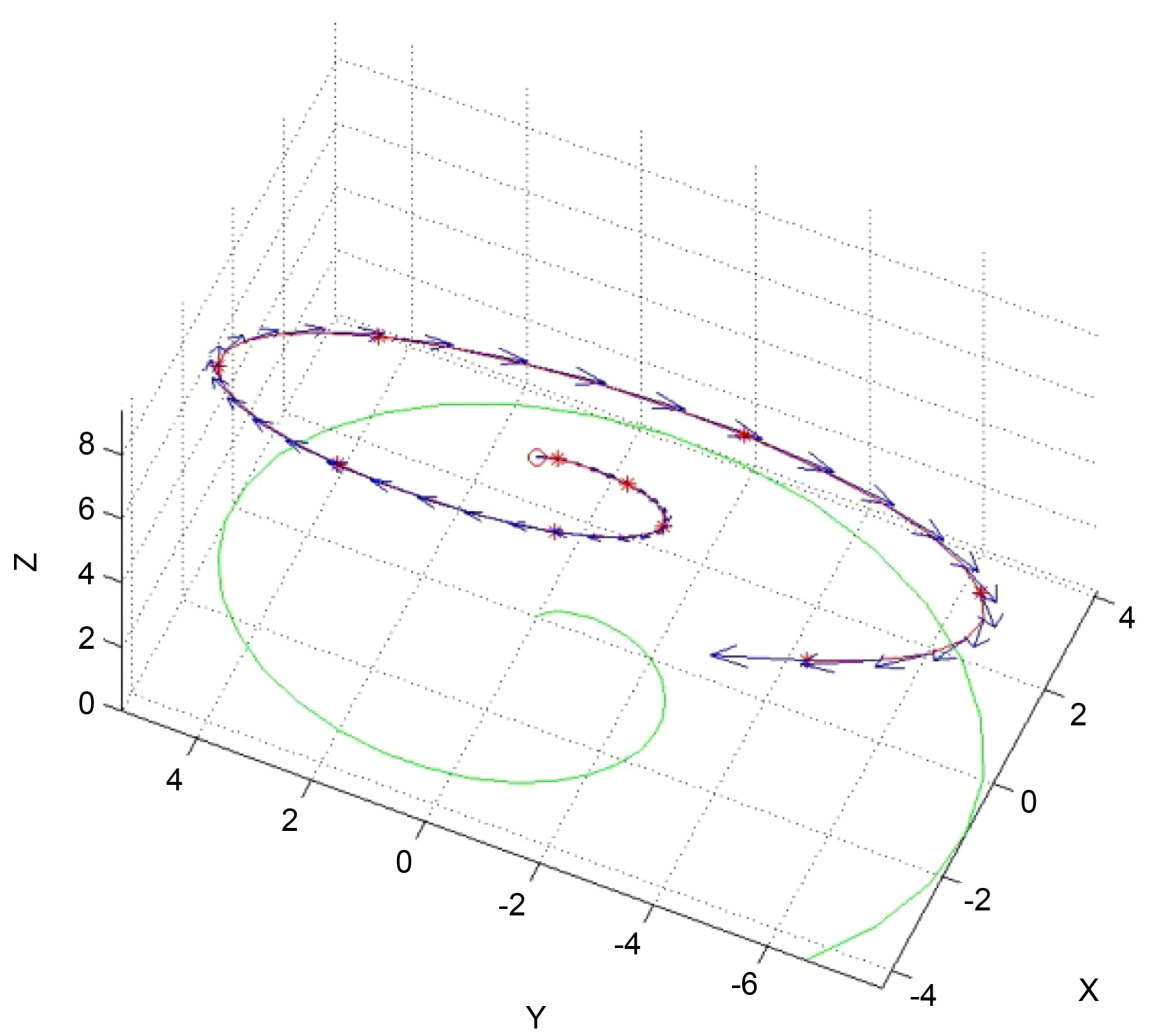

Figure 5. Path obtained by computer, and its projection in the XY plane, in accordance with the parameters of the fourth simulation. Path of the center of mass of a moving object in the hypothetical case that the momentum applied is constant, but the linear translation velocity is variable [53]. 
It should be pointed out in this case, as well as regards those described above, that the path obtained is plane as long as there is no spatial variation in the acting external momentum. The resultant path will always be on the plane defined by the initial linear velocity and the acting torque. Numerous examples of $d y$ namic behavior can be seen in nature that concur with these simulated paths [54].

We can recall a deduction proposed by Dr. Barceló, based on the simulation model obtained, regarding the assumption of constantly accelerated velocity. $\mathrm{He}$ suggests that the reason for the development and growth of helical living bodies, such as conch shells or ammonites, by analogy with the simulation of an accelerated moving object, subjected to a non-coaxial torque, concluding that its shape is also the result of its growth according to a geometric relation, equivalent to the accelerated movement of the dynamic simulation of the Figure 5.

\section{Conclusions, Inventions and Technological Applications}

Finally, we refer to the fact that these conceptual innovations will allow multiple ideas, inventions and technological applications.

Up to this point, we have referred to the main innovations and novel ideas in the theory of dynamic interactions. Due to their importance, I suggest should be part of a new paradigm in mechanics. However, after reading the new book by Dr. Barceló: New paradigm in physics, the reader might possibly find other innovations, proposals and explanations for phenomena in physics, which until now have not been explained.

\section{References}

[1] Barceló, G. (2017) New Paradigm in Physics. Vol. I. This Is the First Book Written by Its Author in English Language. Previously He Had Written Five Other Books Referring to This Same Subject in Spanish Language.

[2] Alejandro, Á.M. and Almudena, M.G. (2016) The Dance of the Spinning Top. Global Journal of Science Frontier Research: A Physics \& Space Science, 16, 15-27. https://globaljournals.org/GJSFR_Volume16/E-Journal_GJSFR_(A)_Vol_16_Issue_ 3.pdf

[3] Barceló, G. (2017) New Paradigm in Physics. Conjectures, Epilogue, Vol. I.

[4] Barceló, G. (2017) New Paradigm in Physics. 21.

[5] Barceló, G. (2017) New Paradigm in Physics. Inconsistency, Epilogue, Vol. I.

[6] Cano, J. (2017) Rotational Dynamics.

[7] See Barceló, G. (2006) The Flight of the Boomerang. Section 11.5-Conjectures on Angular Displacement, Marcombo, Barcelona, 256.

[8] Barceló, G. (2017) New Paradigm in Physics. Chap. 2.2.9.2.

[9] Wikibooks Contributors (2016) Complete Physics Textbook. Wikibooks. https://es.wikibooks.org/w/index.php?title=F\%C3\%ADsica/Texto_completo\&oldid $=241283$

[10] Barceló, G. (2017) New Paradigm in Physics. Chap. 2.2.7-A Study of the Rotation of Bodies.

[11] Barceló, G. (2017) New Paradigm in Physics. Chap. 3, 215. 
[12] Cano, J. (2015) The Pendulum of Dynamic Interactions. Journal of Applied Mathematics and Physics, 3, 1186-1198. http://www.scirp.org/journal/jamp

[13] Barceló, G. (2017) New Paradigm in Physics. Chap. 2.5-Dynamics of the Rotation of Bodies. 168.

[14] Barceló, G. (2017) New Paradigm in Physics. Chap. 2.5-Dynamics of the Rotation of Bodies. 169.

[15] Barceló, G. (2017) New Paradigm in Physics. Chap. 4.8-Comments.

[16] Appell, P. (1941) Traité de MécaniqueRationnelle. Paris.

[17] Barceló, G. (2008) A Rotating World. Chap. 2.4-Resolución Matemática. Marcombo, Barcelona, 41.

[18] Barceló, G. (2017) New Paradigm in Physics. Error Identification, Epilogue, Vol. I. 370.

[19] Barceló, G. (2017) New Paradigm in Physics. Error Identification, Epilogue, Vol. I. 371.

[20] Barceló, G. (2017) New Paradigm in Physics. The Observation of Nature, Epilogue Vol. I.

[21] Barceló, G. (2017) New Paradigm in Physics, 0.3.1-Content.

[22] Barceló, G. (2013) Imago Universi: A Story of the Human Conception of the Cosmos. Arpegio, Barcelona. http://advanceddynamics.net/imago-universi/

[23] Barceló, G. (2013) Proposal of New Criteria for Celestial Mechanics. International Journal of Astronomy and Astrophysics, 3, 385. https://doi.org/10.4236/ijaa.2013.34044

[24] Barceló, G. (2013) Proposal of New Criteria for Celestial Mechanics. International Journal of Astronomy and Astrophysics, 3, 389. https://doi.org/10.4236/ijaa.2013.34044

[25] Barceló, G. (2017) New Paradigm in Physics, Cap. 2.6, 184.

[26] Barceló, G. (2017) New Paradigm in Physics, Cap. 2.6, 185.

[27] Barceló, G. (2017) New Paradigm in Physics. Chap. 2.9.0-Adding angular momenta.

[28] Barceló, G. (2011) Analysis of Dynamics Field Systems Accelerated by Rotation. Dynamics of Non-Inertial Systems. DeMSET-2011 Congress, Miami, USA.

[29] Barceló, G. (2017) New Paradigm in Physics. Amazon, Chap. 3.

[30] Bruhat, G. (1955) Mécanique, Paris.

[31] Barceló, G. (2012) Analysis of Dynamics Fields in No inertial Systems. World Journal of Mechanics, 2, 175-180. http://www.scirp.org/journal/wjm https://doi.org/10.4236/wjm.2012.23021

[32] Barceló, G. (2017) New Paradigm in Physics. Amazon, Figure 4.9.

[33] Barceló, G. (2017) New Paradigm in Physics. Amazon, Chap. 4.1.0.3.

[34] Barceló, G. (2017) New Paradigm in Physics. Amazon, Figure 4.16.

[35] Barceló, G. (2008) A Rotating World. Epigraph 8.2.1, 209. Marcombo, Barcelona. http://dinamicafundacion.com/?page_id $=286$

[36] Bauluz, E. and Barceló, G. (2011) New Dynamic Hypotheses. Video script. Madrid; and Barceló, G. (2017) New Paradigm in Physics. Chap. 4.5.2-Confirmed conceptual model.

[37] Barceló, G. (2017) New Paradigm in Physics. Chap. 4.3.2-Impact of the Interaction 
Torque and the Mathematical Operator.

[38] Barceló, G. (2017) New Paradigm in Physics. Chap. 4.3.0-Mathematical Analysis 275.

[39] Barceló, G. (2006)The Flight of the Boomerang. 280. Marcombo, Barcelona. http://www.dinamicafundacion.com/

[40] Barceló, G. (2017) New Paradigm in Physics. Chap. 4.3.0-Mathematical Analysis. 277.

[41] Barceló, G. (2008) A Rotating World. Section 12.4. Marcombo, Barcelona, 321.

[42] Barceló, G. (2017) New Paradigm in Physics. Chap. 4.3.1—Space Reference.

[43] Barceló, G. (2006)The Flight of the Boomerang. Marcombo, Barcelona, 325.

[44] Barceló, G. (2017) New Paradigm in Physics. Chap. 4.4-Inertial Reactions.

[45] Barceló, G. (2017) New Paradigm in Physics. New Hypotheses. Epilogue, Vol. I.

[46] Barceló, G. (2017) New Paradigm in Physics. The New Equation of Motion. Epilogue, Vol. I.

[47] Barceló, G. (2017) New Paradigm in Physics. Chap. 5.3. Principles and Axioms of Rotational Dynamics. Figure 5.3.

[48] Barceló, G. (2017) New Paradigm in Physics. Chap. 5.4.0-Equation Deduction.

[49] Barceló, G. (2017) New Paradigm in Physics. Chap. 5-General Theory of Mechanics.

[50] Barceló, G. (2017) New Paradigm in Physics. Chap 5. 7-In Search of a New Canon.

[51] Barceló, G. (2017) New Paradigm in Physics. 2.3-Analytical Mechanics. Amazon.

[52] Barceló, G. (2017) New Paradigm in Physics. 5.0.1-Dynamic Axioms. Amazon.

[53] Barceló, G. (2017) New Paradigm in Physics. Figure 5.16.

[54] Barceló, G. (2017) New Paradigm in Physics. 5. 6-Mathematical Simulation. Amazon.

\section{Submit or recommend next manuscript to SCIRP and we will provide best} service for you:

Accepting pre-submission inquiries through Email, Facebook, LinkedIn, Twitter, etc. A wide selection of journals (inclusive of 9 subjects, more than 200 journals)

Providing 24-hour high-quality service

User-friendly online submission system

Fair and swift peer-review system

Efficient typesetting and proofreading procedure

Display of the result of downloads and visits, as well as the number of cited articles

Maximum dissemination of your research work

Submit your manuscript at: http://papersubmission.scirp.org/

Or contactwjim@scirp.org 